Revue européenne des sciences sociales

European Journal of Social Sciences

52-1| 2014

Politiques du libre accès en sciences humaines et sociales

\title{
Dominique Schnapper, 2013, Travailler et aimer. Mémoires, Paris, Odile Jacob, 233 p.
}

Nicolas Sembel

\section{CpenEdition}

\section{Journals}

Electronic version

URL: http://journals.openedition.org/ress/2760

DOI: $10.4000 /$ ress. 2760

ISSN: $1663-4446$

\section{Publisher}

Librairie Droz

\section{Printed version}

Date of publication: 6 May 2014

Number of pages: $299-302$

ISBN: 978-2-600-01829-6

ISSN: 0048-8046

Electronic reference

Nicolas Sembel, « Dominique Schnapper, 2013, Travailler et aimer. Mémoires, Paris, Odile Jacob, 233 p. », Revue européenne des sciences sociales [Online], 52-1 | 2014, Online since 12 May 2014 connection on 22 September 2020. URL : http://journals.openedition.org/ress/2760 ; DOI : https:// doi.org/10.4000/ress. 2760

This text was automatically generated on 22 September 2020

(c) Librairie Droz 


\title{
Dominique Schnapper, 2013, Travailler et aimer. Mémoires, Paris, Odile Jacob, $233 \mathrm{p}$.
}

\author{
Nicolas Sembel
}

1 Dominique Schnapper a choisi de présenter ses mémoires de façon synthétique, en limitant les précisions biographiques; elles ont un faux air de Spectateur engagé (1981, Julliard), l'ouvrage d'entretiens de son père, Raymond Aron, mais produisent un résultat très différent. Avec 230 pages de réponses par courriels aux 60 questions de Sylvie Mesure, judicieusement retranscrites aux côtés de l'entretien (parfois modifié) qu'elle avait accordé à Giovanni Busino - tous les deux qualifiés d' «amis »-, dans la présente revue, en 2006, l'appellation de Mémoires est justifiée. Leur titre, une formule de Freud, guide leur auteure qui "n'évoque jamais le passé de manière consciente » (p. 66) et mobilise le « sentiment de nécessité » (p. 28, p. 215) pesant sur son existence. $\mathrm{Au}$ terme de cette "explicitation de l'implicite ", Dominique Schnapper est allée plus loin peut-être qu'elle ne pouvait l'imaginer ( $«$ je me rends compte rétrospectivement, maintenant que tu me forces à y repenser, que cela a été plus difficile que je ne voulais me l'avouer à moi-même ", p. 62). L'ensemble possède une densité insoupçonnée.

2 En cinq chapitres d'environ quarante pages chacun, découpés chronologiquement (1940-1969, sur la «formation »; 1970-1985, sur "l'après 68 »; 1986-2000, sur les « années fructueuses »; 2001-2012, sur l'École des hautes études en sciences sociales et le Conseil constitutionnel) et une dernière partie sur les thèmes de "l'héritage » et de la «transmission", Dominique Schnapper donne à voir, plus encore que sa personnalité, sa «position paradoxale» (p. 139) dans le «champ intellectuel» (p. 11). Elle dessine non une galerie de portraits mais la structuration d'un univers de relations où les liens faibles et parfois durables jouent un rôle non négligeable (en sociologie, voir p. 69-70, p. 92-93, p. 139-141). Elle affirme à plusieurs reprises sa «marginalité » (p. 139) : «j'ai toujours eu le sentiment que ma place était inexistante» (p. 83); une pique contre la «politique» des comptes rendus de la Revue française de sociologie, exprimée dans l'entretien de 2006, a été supprimée. Cette position se résume à un 
quant-à-soi («ma petite barque personnelle»), expression du parfait accord, inaltérable et couvrant plusieurs décennies, entre des aspirations à une reconnaissance limitée ( «ma vie familiale, mon enseignement et mes travaux me suffisaient pleinement»; «je déteste voyager»; la retraite a été accueillie comme un "soulagement»); et les dispositions d'une intellectuelle "de naissance» («toute l'organisation de la vie familiale tournait autour du travail de mon père »; « il y a une véritable volupté dans l'effort de connaissance, une fois qu'on en a tâté, il est difficile de s'en passer ", p. 206), d'« origine bourgeoise", " fille de », femme de ("c'est mon mariage qui m'a permis de me trouver moi-même», p. 219), de "droite» (p.61) et marquée par "l'ombre de la Shoah» (p. 26, p.112, p. 213). En découlent sa défense intransigeante de la République (elle exprime toutefois une critique interne de la République sur les liens avec la Nation, p. 110-111, ou sur les limites du civisme p.118), et sa critique "externe» (p.181) de la démocratie (par exemple p.170-174). L'« ossature théorique » de l'œuvre (pour une synthèse, voir p. 181-182) est formée par La Communauté des citoyens (1994, Gallimard), La Relation à l'autre (1998, Gallimard), « livre auquel je suis le plus attachée » (p. 71), « essentiel », « auquel je tiens le plus », La Démocratie providentielle (2002, Gallimard) et L'Esprit démocratique des lois (2014, Gallimard), sur « l'idée de corruption des régimes ».

3 Le rapport de Dominique Schnapper à la sociologie se construit autour de deux axes. Le premier regroupe trois "admirations" inégales. D'abord envers "Raymond Aron" (voir le terme de "flambeau», p. 201 et p. 214), même si, "s'agissant de l'héritage paternel, j'ai beaucoup de peine à te répondre. Je me demande si on peut vraiment prendre conscience de cet héritage» (p. 185), et que sa disparition a aussi été une libération «du point de vue intellectuel » (p.95). La toute première question évoque tout de même une sociologie "acceptée ». La relation paternelle est décrite comme une « symbiose intellectuelle et silencieuse »: « je n'ai jamais eu de véritables admirations en dehors de mon père » (p. 218), "pour rien au monde je n'aurais voulu le blesser ", «je ne voulais pas apparaître comme un suiveur aveugle de ses positions, mais je voulais encore moins [...] apporter la moindre nuance à ses analyses, ce qui aurait pu nourrir une attaque du style : "même sa fille pense que..." »(p. 12). Les très aroniennes chroniques publiées dans Aufur et à mesure (2003, Odile Jacob), témoignent de cet héritage ; comme certaines prises de positions conservatrices dans l'ouvrage contre la démocratisation de l'enseignement, l'entrée de la Turquie dans l'Europe ou Stéphane Hessel, qui ne sont pas sans rappeler l'avant-propos de la France de l'intégration (1991, Gallimard).

Admiration envers Bourdieu également, malgré la « rupture » (p. 55), auquel une dette est plusieurs fois exprimée (notamment p. 49-50, p. 74, p. 82, et indirectement, p. 199). Elle se trouve à l'origine de la seule différence revendiquée avec le père, autour de son " rapport aux Classiques ", qui détermine un rapport à la sociologie "très différent " (p. 168, et également p. 53). «J'ai abordé la sociologie par le bas, si l'on peut dire, en réalisant l'enquête à Bologne, L'Italie rouge et noire (Thèse, publiée en $1971 \mathrm{chez}$ Gallimard), puis celle sur le public des musées, avant toute formation générale à la discipline. J'ai d'abord pratiqué l'enquête et lu les premières publications de Pierre Bourdieu avant les auteurs qui ont fondé la pensée sociologique. C'est peut-être la raison pour laquelle je n'ai jamais vraiment travaillé ni compris les œuvres d'Auguste Comte, de Pareto et de Marx ». Une critique de la sociologie empirique américaine, exprimée dans l'entretien de 2006, a été supprimée. 
Cependant, Durkheim et la sociologie scientifique ou Weber et la sociologie compréhensive n'en font pas moins partie de son "panthéon ». Et surtout Tocqueville, moins sociologue que politiste, objet d'une "admiration sans borne» (p.122). La politique et ses contraintes, l'activité, la prise de parti quotidienne, et l'idéologie, sont toutes loin de la science, mais «l'ombre de la Shoah » les rend nécessaires.

Un deuxième axe, découlant du premier, soumet ces «Classiques », et toute théorie, à la "méthode " (voir La Compréhension sociologique, 2012 [1999], PUF), qui fonde le métier de sociologue, « recherche honnête de la vérité grâce au travail et à la réflexion ", lequel travail constitue un «bien commun» (p. 202) qu'il s'agit de défendre sur la base de "convictions de chercheur " contre "la bureaucratisation de la recherche " (p. 141) et l'évaluation ( « la seule évaluation quantitative de la production intellectuelle me paraît dramatique ", p. 161), au nom du terrain comme « charbon », de la « véritable tradition» sociologique, de l'« entre-deux» macro/micro (p.166), ou de l'analyse typologique.

7 De la sociologisation de la philosophie à la politisation de la sociologie, l'itinéraire de Dominique Schnapper reste unique du fait de sa position spécifique originelle. Elle se définit comme le "contraire d'une historienne des idées politiques ", a des mots très durs, au nom de la rigueur scientifique, pour quatre institutions républicaines: la khâgne de philosophie (« cette philosophie-là était un pur verbalisme [...] elle n'était pas un véritable projet intellectuel»; plus loin elle évoque « les philosophes politiques, qui risquent toujours de devenir normatifs", et dont la «seule connaissance " est « intuitive », «à partir de leur expérience sociale», p. 106) ; Sciences Po (« du point de vue intellectuel, j'ai été déçue», notamment par des élèves "dépourvus de toute ambition intellectuelle»; sans compter le sexisme le plus choquant, p.172); l'Université ( de plus en plus, les universitaires sont tentés de choisir des médiocres »); et le Conseil constitutionnel («j'ai souffert du mépris ou de l'ignorance des valeurs intellectuelles qui régnaient alors à SciencesPo-sentiment que j'ai à nouveau ressenti, même si c'était sous une forme très courtoise, au Conseil constitutionnel »; «Ils parlaient du livre de Dominique de Villepin sur les Cent-Jours comme d'un livre d'histoire et ils ignoraient jusqu'au nom des plus grandes gloires du monde intellectuel »; «j'étais une sociologue "comestible" », qualificatif figurant dans l'entretien de 2006 mais supprimé dans le livre). Ce qui n'altère pas le profond respect de l'auteure pour, respectivement, la philosophie, la politique, les intellectuels et le droit.

8 Privé et public sont entremêlés (voir par exemple p. 210). De manière anecdotique, une pure coïncidence de dates rapproche, en septembre 1958, la naissance de la Licence de sociologie, sous l'impulsion de Raymond Aron, et la « prise de conscience » de l'histoire d'amour avec Antoine Schnapper, à Rome («c'était une révélation de ce qui s'était construit dans l'inconscient au cours des mois précédents "; " c'est ainsi que se joue une vie», p. 27 et p. 220), époux dépeint, jusqu'au "portrait sociologique» final, comme une sorte de double social. Ainsi, l'origine (privée) du «choix» des objets d'étude (publics), est-elle l'«illustration du lien entre les expériences sociales du chercheur et son projet sociologique»(p.176). La sociologie offre l'occasion de construire une distance critique à ses propres particularismes, sorte de neutralisation culturelle, qui rejoint peut-être le « relativisme culturel relatif », auquel D. Schnapper "adhère totalement " (p.118), et fonde la condition de possibilité des «émotions" 
collectives, de l'«élan» pouvant dynamiser de façon pleinement démocratique une communauté de citoyens.

9 Reste dès lors la dernière piste, jusqu'ici « négligée ", « à tort » et à regret (p. 175-177), «je fais le projet de l'aborder»: plus encore que l'interrogation (notamment « par le bas ", p. 24 et p. 86) sur la judéité, le retour sur, selon nous, la véritable " communauté historique » (p. 81) (définie comme « histoire partagée, à la fois réelle et imaginaire ») de Dominique Schnapper. Femme «tout court», universelle, qui critique René Rémond - sous l'autorité duquel on peut « discuter gravement », à Sciences Po en 1953, sur le point de savoir si les femmes devaient avoir le droit de vote ( $"$ J'en ai été ahurie et je le suis encore ») -, ou s'afflige des réactions « ahurissantes » au comportement de « DSK » (Dominique Strauss-Kahn) presque soixante ans plus tard, en 2011. L'étude de ce « fonds anthropologique, très profond», « noyau dur culturel» (p. 178) du sexisme, pourrait ainsi parachever sa quête socio-analytique de l'« essentiel»: «comment les hommes renouvellent chaque jour le sens qu'ils donnent à leur destin social » (p. 79).

\section{AUTHORS}

\section{NICOLAS SEMBEL}

Université de Bordeaux, ESPE - Centre Émile Durkheim 